\title{
Parity does not affect diabetes complications in women with type 1 diabetes
}

\author{
Katarzyna Cypryk', Joanna Grycewicz², Patrycja Swierzewska³, Marcin Kosinski', \\ Andrzej Lewinski ${ }^{4}$, Monika Zurawska-Klis ${ }^{1}$ \\ ${ }^{1}$ Department of Diabetology and Metabolic Diseases, Medical University of Lodz, Poland \\ ${ }^{2}$ Regional Centre of Occupational Medicine, Prevention and Treatment Centre in Lodz, Poland \\ ${ }^{3}$ Outpatient Department of Diabetology in the Polish Mother's Memorial Hospital/Research Institute in Lodz, Poland \\ ${ }^{4}$ Department of Endocrinology and Metabolic Diseases, Medical University of Lodz, Poland
}

Cypryk K, Grycewicz J, Swierzewska P, Kosinski M, Lewinski A, Zurawska-Klis M. Parity does not affect diabetes complications in women with type 1 diabetes. Ann Agric Environ Med. 2018; 25(1): 114-119. doi: 10.5604/12321966.1230738

\section{Abstract}

Introduction. The problem concerning the impact of pregnancy on diabetic complications is a matter for discussion as there is some evidence suggesting that pregnancy may trigger development or progression of diabetic chronic complications. However, currently available data concerning this issue is still controversial.

Objective. The aim of the study was to evaluate the impact of obstetric history on the development of chronic microangiopatic and macroangiopatic complications in type 1 diabetic women.

Materials and Method. The retrospective study comprised 226 white Caucasian type 1 diabetic women, including 190 parous and 36 nulliparous women. Anthropometric data, information concerning the course of the disease, including metabolic control and chronic complications, together with obstetric history, were registered.

Results. Parous women were older $(p<0.001)$, but did not differ significantly regarding metabolic control in the course of the disease $(p>0.05)$ and diabetes duration $(p>0.05)$ from nulliparous subjects. There were no significant differences in the incidence $(p>0.05)$ nor onset $(p>0.05)$ of chronic diabetes complications between the groups. The number of deliveries did not correlate with either the incidence nor the onset of chronic complications. Longer DM duration at the moment of first delivery was related to the higher incidence of retinopathy $(p<0.01)$, nephropathy $(p<0.05)$ and neuropathy $(p<0.001)$.

Conclusions. The incidence of chronic diabetic complications does not differ between parous women and the subjects that were never pregnant, and is not related to the number of pregnancies.

\section{Key words}

diabetes, obstetric history, chronic complications

\section{INTRODUCTION}

The issue concerning the impact of pregnancy on diabetic complications remains under constant debate. The problem is of great importance and should be thoroughly discussed and considered by women planning childbirth. Current data assessing this matter in a complex, multifactorial and multidirectional way, is limited. The available literature concerns mainly retinopathy [1-14] and nephropathy [2, 11, 14-19], while the issue of neuropathy and macrovascular diseases is poorly documented $[2,11]$. Moreover, the shortterm impact of pregnancy on diabetic complications have been widely described, while data on diabetic complications in the longer perspective is still lacking. Publications on this topic are focused on neonatal outcomes rather than aspects of maternal wellbeing. What is more, most of the literature regarding this issue was published quite a long time ago [4, $5,7,9,10,11,14,15,16,18]$ when the available medical care, targets of metabolic control and treatment options were very different. Additionally, childbirth planning was rather rare as its importance was not highlighted sufficiently. Moreover, the results found by the authors in the literature are not consistent $[7,9,11,12,14,16,17]$. The main questions raised by diabetic women contemplating childbirth are:

Address for correspondence: Monika Zurawska-Klis, Medical University of Lodz, Department of Diabetology and Metabolic Diseases, Poland

E-mail: moniazur@wp.p I

Received: 18 October 2015; accepted: 13 April 2016; first published on January 2017
- Does the pregnancy itself affect the development of a certain complication?

- Can it deteriorate the prognosis of an already existing complication?

- Does the moment of conception matter?

It is suggested that some factors resulting from pregnancy physiology, such as fluid retention, increased cardiac output, vasodilation, and hypercoagulation, may affect capillary bed autoregulation in a way that is observed in the initial steps of microangiopathic complications development [3]. Despite the controversies, it seems that pregnancy may rather worsen the complication that is already present at conception than increase the risk of its development $[1,2,9,10,16]$. Moreover, remission is often observed after delivery $[3,9]$. Therefore, it was decided to perform a retrospective study assessing the long-term influence of pregnancy(ies) on the incidence of diabetes chronic complications.

\section{OBJECTIVE}

The aim of the study was to assess the long-term influence of pregnancy(ies) on the incidence of diabetes chronic complications. 


\section{MATERIALS AND METHOD}

The retrospective observation comprised 232 Caucasian white women suffering from type 1 diabetes mellitus (T1DM). The participants in the study were recruited from the Outpatient Department of Diabetology of the Polish Mother's Memorial Hospital/Research Institute in Lodz, Poland, between January 2010 - December 2012. 190 women had completed at least one successful pregnancy, with delivery $\geq 22$ weeks of gestation (parous women - group P), and the remaining 36 were never pregnant, independent of the reason (nulliparous women group NP). The remaining 6 women were excluded from the initial study sample because they had had only miscarriages before the $22^{\text {nd }}$ gestational week, and had no children.

Basic data such as age, weight and height was taken, and blood pressure measured at the moment of data collection. Data regarding obstetric history was also recorded: number of pregnancies and deliveries, year of subsequent labours and mode of delivery. Results of laboratory tests were gathered, including $\mathrm{HbA}_{1 \mathrm{c}}$ (every available result), last lipids and creatinine concentrations. Information concerning chronic diabetes complications was also taken, including the presence and grade of complications and the onset of diagnosis. Diabetic retinopathy was classified by experienced ophthalmologists as background or proliferative. Nephropathy was classified as microalbuminuria, overt proteinuria or renal insufficiency. Neuropathy was diagnosed by a diabetologist on the basis of clinical symptoms together with neurologic examination. History of hypertension, coronary heart disease, peripheral artery disease, amputation and diabetic foot was also taken. Information on smoking was recorded. All the necessary information was obtained from the patient's thorough medical history and available medical records, as well as from the database of the Outpatient Department of Diabetology.

Metabolic control was assessed in 3 time intervals: 1) mean $\mathrm{HbA}_{1 \mathrm{c}}$ from the first year of the disease, 2) mean $\mathrm{HbA}_{1 \mathrm{c}}$ from subsequent pregnancies, and 3) mean $\mathrm{HbA}_{1 \mathrm{c}}$ from the whole course of the disease as an index of metabolic control (calculated from all available $\mathrm{HbA}_{1 \mathrm{c}}$ results).

Normality tests were performed on all the data and appropriate statistical test were applied. Data with normal distribution were compared using an unpaired, two-tailed Student's t-test, and data with not normal distribution, as well as the categorical data, were compared using the Chi-square test. Spearman's correlation coefficient was calculated in the case of normal distribution, and the Pearson correlation coefficient calculated in the case of non- normal distribution. Factors that were significantly associated with consecutive complications in univariate analysis were then included as covariates in the multivariate logistic regression analysis. $P$ values $<0.05$ were considered statistically significant. All the statistical analyses were performed with the PQ STAT software package (version 1.4.4 for Windows, License No. 01500256).

The study protocol was approved by the local Ethics Committee at the Medical University of Lodz.

\section{RESULTS}

Whole study population. Clinical characteristics of the study population are presented in Table 1 . The women from group P were older $(p<0.001)$, had higher BMI $(p<0.01)$ and were
Table 1. Characteristic of the study population

\begin{tabular}{lccc}
\hline Parameter & $\begin{array}{c}\text { Group P } \\
(\mathrm{n}=190)\end{array}$ & $\begin{array}{c}\text { Group NP } \\
(\mathrm{n}=36)\end{array}$ & $\boldsymbol{p}$ \\
\hline Age (years) & $38.5 \pm 7.9$ & $32.5 \pm 8.4$ & 0.000051 \\
\hline Age at diagnosis (years) & $19.6 \pm 9.0$ & $15.8 \pm 7.4$ & 0.0199 \\
\hline DM duration (years) & $18.9 \pm 8.3$ & $16.6 \pm 7.7$ & 0.127 \\
\hline Weight $(\mathrm{kg})$ & $66.72 \pm 10.73$ & $62.01 \pm 11.04$ & 0.016 \\
\hline BMl $\left(\mathrm{kg} / \mathrm{m}^{2}\right)$ & $24.77 \pm 3.64$ & $22.96 \pm 3.86$ & 0.007 \\
\hline HbA $(\%)$ & $7.54 \pm 1.22$ & $7.74 \pm 1.57$ & 0.47 \\
\hline Total cholesterol (mg/dl) & $194.29 \pm 35.6$ & $163 \pm 45.03$ & 0.0003 \\
\hline Triglycerides $(\mathrm{mg} / \mathrm{dl})$ & $87.11 \pm 41.54$ & $77.65 \pm 39.52$ & 0.32 \\
\hline HDL-cholsterol $(\mathrm{mg} / \mathrm{dl})$ & $70.93 \pm 17.91$ & $63.81 \pm 24.01$ & 0.11 \\
\hline LDL-cholesterol $(\mathrm{mg} / \mathrm{dl})$ & $106.87 \pm 33.36$ & $81.54 \pm 26.53$ & 0.0013 \\
\hline SBP $(\mathrm{mmHg})$ & $122.92 \pm 16.2$ & $121.94 \pm 14.36$ & 0.73 \\
\hline DBP $(\mathrm{mmHg})$ & $77.77 \pm 9.35$ & $75.69 \pm 9.02$ & 0.22 \\
\hline Creatinine Clearance $(\mathrm{ml} / \mathrm{min})$ & $113.62 \pm 40.35$ & $110.5 \pm 34.58$ & 0.7 \\
\hline Smoking Yes/No & $14 / 176$ & $5 / 31$ & 0.33 \\
\hline
\end{tabular}

diagnosed with DM later $(p<0.05)$, compared with the women from group NP. However, it must be stressed that neither $\mathrm{DM}$ duration nor mean metabolic control in the course of the disease (expressed as the average $\mathrm{HbA}_{1 \mathrm{c}}$ ) differed significantly between the groups. There was no significant difference in smoking frequency between both groups $(p=0.19)$. There were also no significant differences in the incidence of complications between both groups, except for the rate of ischaemic heart disease (Tab. 2). Moreover, the onset of complications was not affected by the history of pregnancy in the past (data not shown).

Table 2. Incidence of complications in the groups

\begin{tabular}{lccc}
\hline Parameter & $\begin{array}{c}\text { Group P } \\
(\mathrm{n}=190)\end{array}$ & $\begin{array}{c}\text { Group NP } \\
(\mathrm{n}=36)\end{array}$ & $p$ \\
\hline Background retinopathy & 82 & 11 & 0.2 \\
\hline Proliferative retinopathy & 12 & 2 & 0.89 \\
\hline Microalbuminuria & 58 & 8 & 0.36 \\
\hline Proteinuria & 11 & 3 & 0.52 \\
\hline Renal Insufficiency & 6 & 2 & 0.45 \\
\hline Neuropathy & 41 & 11 & 0.2 \\
\hline Hypertension & 36 & 3 & 0.13 \\
\hline Ischaemic heart disease & 0 & 1 & 0.019 \\
\hline Peripheral artery disease / Amputation & $1 / 1$ & $1 / 1$ & 0.17 \\
\hline Diabetic foot & 6 & 1 & 0.92 \\
\hline
\end{tabular}

While considering the relationships between certain complications and their potential risk factors, it was noted that DM duration and age were the strongest predictors (Tab. 3). Additionally, mean $\mathrm{HbA}_{1 \mathrm{c}}$ from the first year of the disease correlated only with the incidence of background retinopathy $(\mathrm{r}=0.15 ; p<0.05)$, microalbuminuria $(\mathrm{r}=0.16$; $p<0.05)$, and hypertension $(\mathrm{r}=0.18, p<0.01)$. No relationship was observed between the basic parameters and the onset of complications (data not shown).

Multivariate logistic regression analysis was then performed, with the subsequent complications as dependent variable. Age was shown as a predictor for only microalbuminuria $(\mathrm{OR}=0.82 ; 95 \% \mathrm{CI}<0.71 ; 0.95>$; $p=0.01)$. 
Table 3. Relationship between basic parameters and chronic complications in the whole group

\begin{tabular}{|c|c|c|c|c|c|c|c|c|c|c|c|c|}
\hline Parameter & 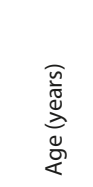 & 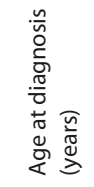 & 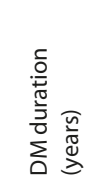 & 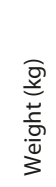 & 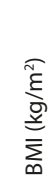 & 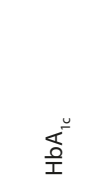 & $\begin{array}{l}\widehat{\bar{े}} \\
\text { ठे } \\
\text { छે } \\
\cup\end{array}$ & $\begin{array}{l}\widehat{\bar{े}} \\
\text { ठे } \\
\text { छે } \\
\text { ৩ }\end{array}$ & $\begin{array}{l}\widehat{\bar{े}} \\
\text { ठे } \\
\text { छे } \\
\text { 모 }\end{array}$ & $\begin{array}{l}\overline{\bar{े}} \\
\text { oे } \\
\text { है } \\
\vec{\jmath}\end{array}$ & 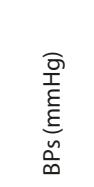 & $\begin{array}{l}\text { ठิ } \\
\text { I } \\
\text { है } \\
\text { है } \\
0 \\
0 \\
0\end{array}$ \\
\hline Background retinopathy & $0.34 * * *$ & $-0.16^{*}$ & $0.5^{*}$ & NS & NS & $0.19^{* *}$ & NS & NS & NS & NS & $0.24^{* * *}$ & $0.18^{* *}$ \\
\hline Proliferative retinopathy & $0.21^{* *}$ & $-0.21^{*}$ & $0.44^{* * *}$ & NS & NS & NS & NS & NS & NS & NS & $0.22^{* * *}$ & NS \\
\hline Microalbuminuria & $0.16^{*}$ & NS & $0.28^{* * *}$ & NS & NS & $0.23^{* * *}$ & NS & NS & NS & NS & $0.26^{* * *}$ & $0.16^{*}$ \\
\hline Proteinuria & NS & $-0.27^{* *}$ & $0.28^{* * *}$ & NS & NS & NS & $0.22^{*}$ & NS & NS & NS & NS & NS \\
\hline Renal Insufficiency & NS & $-0.2^{*}$ & $0.29 * * *$ & NS & NS & NS & NS & NS & NS & NS & $0.17^{* *}$ & NS \\
\hline Neuropathy & $0.26^{* * *}$ & $-0.16^{*}$ & $0.44^{* * *}$ & NS & NS & $0.24^{* * *}$ & NS & NS & NS & NS & $0.13^{* *}$ & NS \\
\hline Hypertension & $0.34^{* * *}$ & NS & $0.36^{* * *}$ & NS & NS & $0.16^{*}$ & $0.21^{*}$ & NS & NS & NS & - & - \\
\hline Ischaemic heart disease & NS & NS & NS & NS & NS & NS & NS & NS & NS & NS & NS & NS \\
\hline Diabetic foot & $0.2^{*}$ & NS & $0.14^{*}$ & NS & NS & NS & NS & NS & NS & NS & NS & NS \\
\hline
\end{tabular}

$<0.05 ;{ }^{*} p<0.05 ;{ }^{* *} p<0.01 ;{ }^{* * *} p<0.001$

DM duration qualified as a predictor for background retinopathy $(\mathrm{OR}=1.15 ; 95 \% \mathrm{CI}<1.08 ; 1.23>; p=0.000011)$, proliferative retinopathy $(\mathrm{OR}=1.33 ; 95 \% \mathrm{CI}<1.12 ; 1.57>$; $p=0.00067)$, microalbuminuria $(\mathrm{OR}=1.06 ; 95 \% \mathrm{CI}<1.01 ; 1.11>$; $p=0.016)$, proteinuria $(\mathrm{OR}=1.24 ; 95 \% \mathrm{CI}<1.08 ; 1.43>; p=0.001)$, renal insufficiency $(\mathrm{OR}=1.32 ; 95 \% \mathrm{CI}<1.09 ; 1.60>; p=0.003)$, and neuropathy $(\mathrm{OR}=1.14 ; 95 \% \mathrm{CI}<1.07 ; 1.22>$; $p=0.000017)$, while $\mathrm{HbA}_{1 \mathrm{c}}$ was revealed as a predictor for neuropathy $(\mathrm{OR}=1.40 ; 95 \% \mathrm{CI}<1.01 ; 1.94>; p=0.038)$. Correlation between systolic blood pressure and proliferative retinopathy $(\mathrm{OR}=1.06 ; 95 \% \mathrm{CI}<1.00 ; 1.13>; p=0.027)$ as well as between total cholesterol concentration and proteinuria $(\mathrm{OR}=1.02$; $95 \% \mathrm{CI}<1.00 ; 1.04>; p=0.024)$ were also disclosed. History of pregnancy did not predict any complication in multivariate logistic analysis. No significant associations were found regarding macroangiopatic complications.

Parous women. No significant relationship was found between parity and the rate of chronic diabetes complications, with exception of ischaemic heart disease (Tab. 4). Longer $\mathrm{DM}$ duration at the moment of first delivery was related to the higher rate of almost all diabetes chronic complications, especially concerning background retinopathy (Tab. 5). As for the relation of age at first delivery, the only significant positive correlation was found with background retinopathy

Table 4. Relationship between pregnancy and incidence of complications

\begin{tabular}{lccc}
\hline Parameter & $\mathrm{r}$ & $\mathrm{Cl}$ & $p$ \\
\hline Background retinopathy & 0.08 & $\leq-0.04 ; 0.21>$ & 0.17 \\
\hline Proliferative retinopathy & 0.01 & $\leq-0.12 ; 0.14>$ & 0.85 \\
\hline Microalbuminuria & 0.06 & $\leq-0.07 ; 0.19>$ & 0.34 \\
\hline Proteinuria & -0.04 & $\leq-0.17 ; 0.9>$ & 0.56 \\
\hline Renal Insufficiency & -0.04 & $\leq-0.18 ; 0.08>$ & 0.47 \\
\hline Neuropathy & -0.08 & $\leq-0.21 ; 0.04<>$ & 0.21 \\
\hline Hypertension & 0.1 & $\leq-0.03 ; 0.22>$ & 0.12 \\
\hline Ischaemic heart disease & - & - & - \\
\hline Peripheral artery disease / Amputation & -0.08 & $\leq-0.21 ; 0.04>$ & 0.18 \\
\hline Diabetic foot & 0.008 & $\leq-0.12 ; 0.13>$ & 0.9 \\
\hline
\end{tabular}

Data presented as Pearson correlation coefficient
Table 5. DM duration at first delivery in relation to complications

\begin{tabular}{lccc}
\hline Parameter & $r$ & $\mathrm{Cl}$ & $p$ \\
\hline Background retinopathy & 0.36 & $\leq 0.20 ; 0.50>$ & 0.000016 \\
\hline Proliferative retinopathy & 0.24 & $\leq 0.07 ; 0.40>$ & 0.004 \\
\hline Microalbuminuria & 0.21 & $\underline{\leq 0.03 ; 0.37>}$ & 0.013 \\
\hline Proteinuria & 0.24 & $\underline{\leq 0.07 ; 0.40>}$ & 0.003 \\
\hline Renal Insufficiency & 0.27 & $\underline{\leq 0.10 ; 0.42>}$ & 0.0014 \\
\hline Neuropathy & 0.3 & $\leq 0.14 ; 0.45>$ & 0.00029 \\
\hline Hypertension & 0.12 & $\leq 0.05 ; 0.29>$ & 0.15 \\
\hline
\end{tabular}

Ischaemic heart disease

Peripheral artery disease / Amputation

\begin{tabular}{llll}
\hline Diabetic foot & $0.006 \quad \leq 0.16 ; 0.17>$ & 0.94
\end{tabular}

Data presented as Pearson correlation coefficient

and diabetic foot (data not shown). Similarly, the number of pregnancies had no impact on the rate of chronic DM complications (data not shown).

DM duration was related to all the complications with the exception of diabetic foot. A significant negative relationship was also observed between the age at DM diagnosis and the incidence of retinopathy, proteinuria, renal insufficiency, and neuropathy in this group. There was no significant correlation between triglycerides and LDL-cholesterol concentration and the rate of chronic complications. Body weight correlated only with proliferative retinopathy $(\mathrm{r}=0.17 ; p<0.05)$. Total cholesterol was significantly related to the incidence of proteinuria $(\mathrm{r}=0.23 ; p<0.01)$ and hypertension $(\mathrm{r}=0.18$; $p<0.05)$ while HDL-cholesterol correlated with proteinuria $(\mathrm{r}=0.21 ; p<0.05)$. Systolic blood pressure correlated with the rate of both background and proliferative retinopathy $(\mathrm{r}=0.25 ; p<0.001$ and $\mathrm{r}=0.26 ; p<0.001$, respectively), microalbuminuria $(\mathrm{r}=0.27 ; p<0.001)$, proteinuria $(\mathrm{r}=0.14$; $p<0.05)$, renal insufficiency $(\mathrm{r}=0.21 ; p<0.01)$ and neuropathy $(\mathrm{r}=0.17 ; p<0.05)$. A significant relationship was also found between diastolic blood pressure and incidence of background retinopathy $(\mathrm{r}=0.17 ; p<0.05)$ and microalbuminuria $(\mathrm{r}=0.15$; $p<0.05)$. Mean $\mathrm{HbA}_{1 c}$ from the course of the disease was related significantly to the rate of the background retinopathy $(\mathrm{r}=0.19$; $p<0.01)$, microalbuminuria $(\mathrm{r}=0.22 ; p<0.01)$, neuropathy 
$(\mathrm{r}=0.21 ; p<0.01)$ and hypertension $(\mathrm{r}=0.15 ; p<0.05)$, while the mean $\mathrm{HbA}_{1 \mathrm{c}}$ from the first year of the disease had no impact on the incidence of complications. Similarly, mean $\mathrm{HbA}_{1}$ from the course of subsequent pregnancies was not related to the rate of complications (data not shown).

Nulliparous women. In the subgroup of NP women, DM duration was related to the incidence of all the complications $(p<0.05)$, but not nephropathy. Age, age at DM diagnosis, weight, BMI, total cholesterol, triglycerides, HDL-cholesterol, LDL-cholesterol and blood pressure affected neither the incidence nor the onset of complications. Similarly, no correlation was observed between the mean $\mathrm{HbA}_{1 \mathrm{c}}$ from the first year of the disease and the rate of chronic DM complications (data not shown).

\section{DISCUSSION}

Available data regarding the impact of obstetric history on chronic diabetes complication in the long-term perspective is limited. The women observed in this study had been, on average, $16.76 \pm 7.76$ years under medical care, and were, on average, $18.5 \pm 6.7$ years after the first delivery. The results of this study show that the history of pregnancy and increasing parity did not affect the long-term risk for diabetic complications. Moreover, it did not accelerate the development of complications. No significant differences were observed in the incidence of complications between the analyzed groups, except for the rate of ischaemic heart disease. However, it should be stressed that this complication was noted in only one subject from the group NP, it therefore seems that no general conclusion should be drawn on the basis of this statistical result. Mean $\mathrm{HbA}_{1 c}$ from the course of subsequent pregnancies in the parous women did not affect significantly the rate of complications. On the basis of the presented results, it also seems that DM duration at the moment of first pregnancy is of great importance. This was also noted in earlier studies where DM duration at the moment of becoming pregnant was predictive of the presence and severity and progression of retinopathy during pregnancy [4]. Therefore, early motherhood seems to be protective in terms of diabetic complications.

Retinopathy. Some evidence suggest that diabetic retinopathy may advance during pregnancy [5]. However, this effect seems to be short-term, with an improvement noted at post-partum [6]. Progression from non-proliferative to proliferative retinopathy varies and was noted in up to $30 \%$ of subjects and required laser photocoagulation [7]. Temple et al. prospectively studied 179 pregnancies in 139 T1DM women. Progression was observed in 5\% of cases and its rate was significantly higher in patients with longer DM duration, as well as in women with moderate to severe background retinopathy at first visit. Initial $\mathrm{HbA}_{1 c}$ was higher, and after 24 weeks its reduction was greater in women experiencing progression of retinopathy, but these changes did not reach statistical significance [8]. Prospective analysis by Moloney et al. revealed that pregnancy was associated with both the development and progression of retinopathy. While 6 months post-partum, the background changes had regressed to control levels, regression of proliferative changes was not complete. In this study, similar to those in the current study, DM duration was a risk factor for the development and progression of retinopathy [9]. Other authors have observed a slight progression during gestation of both background retinopathy (16\%) and proliferative retinopathy after laser photocoagulation before pregnancy (16\%). On the contrary, progression was seen in $86 \%$ of women with proliferative retinopathy untreated prior to pregnancy and further deterioration was protected by laser treatment during pregnancy. DM duration, similar to the presented results, was positively related to progressive proliferative diabetic retinopathy, independently of glucose control [10].

There is also some evidence suggesting that pregnancy may rather worsen than induce retinopathy, and even in the case of progression it tends to remit after delivery. The results of the DCCT showed that pregnancy was related to more rapid progression of retinopathy for one year after delivery, with no long-term deleterious effect of pregnancy in the group of women with short diabetes duration [6]. Hemachandra et al. compared women who had completed at least one successful pregnancy and well-matched women who had not conceived. In multivariate analysis, pregnancy in the history predicted only neuropathy incidence and no other complication. The authors conclude that pregnancy in IDDM women does not increase the risk of diabetes complications in the long-term. In the short-term, however, it may accelerate the development of some complications, such as neuropathy [11]. Similarly, Proniewska-Skretek et al. observed that pregnancy has no significant impact on the progression of diabetic retinopathy diagnosed before conception [12]. Another prospective study aimed at evaluation of the progression of retinopathy during pregnancy and postpartum. The model of multivariable logistic regression analysis showed that nulliparity was the only predictor for retinopathy severity assessed in the third trimester [1]. On the other hand, regression of retinopathy may be also noted during pregnancy. Rasmussen et al. surprisingly observed regression during pregnancy in 2 women with type 2 diabetes with macular oedema present in early pregnancy [13]. Moreover, a cross-sectional study on 28 women suggested a lower rate of retinopathy progression in the long-term in parous women (19\%), compared to nulliparous subjects (50\%) [14]. Therefore, similar to the presented results, available data suggest that pregnancy itself is not a risk factor for developing retinopathy in the long perspective. Like some other authors, the authors of the current study have also noted a significant correlation between age, DM duration, age at diagnosis, average $\mathrm{HbA}_{1 \mathrm{c}}$, blood pressure and the incidence of retinopathy. In multivariable logistic regression analysis however, only DM duration and systolic blood pressure, but not history of pregnancy, qualified as predictors for retinopathy.

Nephropathy. Diabetic nephropathy accompanies about $5 \%$ of pregnancies in diabetic women, especially those with type 1 diabetes. Its progression is characterized by tendency to hypertension and decrease in glomerular filtrations rate, which can be attenuated by proper treatment of hypertension and tight glycaemic control [15]. Deterioration of pre-existing proteinuria may be also noted with the greatest increase observed in subjects with higher proteinuria at conception [16]. Young et al. observed 43 pregnant diabetic women with or without prepregnancy nephropathy. Increase was noted in serum creatinine one year postpartum, compared to the first trimester in the subjects with nephropathy. No 
significant difference in urinary albumin excretion, serum creatinine and creatinine clearance one year postpartum when compared to prepregnancy was observed between the groups. However, in the women with pre-existing nephropathy, a higher incidence of chronic hypertension, preeclampsia and lower gestational age at birth was observed. Therefore, the authors claim that pregnancy was not associated with development and progression of diabetic nephropathy in subjects with or without mild nephropathy. The presence of diabetic nephropathy, however, was related to an increased risk of perinatal complications [17]. Similarly, the study by Miodovnik et al. showed that pregnancy and increasing parity affected neither the risk for development of nephropathy nor accelerated progression of renal disease in women with preexisting nephropathy. The authors observed that proteinuria appearing during pregnancy, as well as glycaemic control during pregnancy, had significant impact on the subsequent development of nephropathy [18]. In the current study, however, mean $\mathrm{HbA}_{1 c}$ from subsequent pregnancies had no influence on the rate of any complications in the long-term. Hemachandra et al. in their study have disclosed that parous women were not at an increased risk of nephropathy later in life [11]. The same conclusion comes from an observational study on a group of 93 women, where 26 women became pregnant and delivered during the followup period. It was revealed that pregnancy had no adverse long-term impact on kidney function and survival in type 1 diabetic women with nephropathy and well-preserved kidney function at conception. It should be stressed, however, that the population of women who became pregnant in this study was significantly younger, with also a significantly shorter duration of diabetes. The short-term influence of pregnancy on blood pressure and proteinuria was not evaluated in this study as the data from available records were incomplete [19]. The results of the presented study remain in line with these observations as no significant long-term impact of obstetric history on the incidence of nephropathy were noted. The risk for nephropathy correlated only with age, $\mathrm{DM}$ duration, mean $\mathrm{HbA}_{1 \mathrm{c}}$ in the course of diabetes and blood pressure. Logistic regression analysis further confirmed the relationship between both age and DM duration and the incidence of nephropathy.

Neuropathy. The impact of pregnancy on the risk of neuropathy was not sufficiently studied and available data is limited to only a few papers. In the short-term, it seems that pregnancy may accelerate its development, but this effect seems to be transient. The Pittsburgh Epidemiology of Diabetes Complications Study showed that subjects who had delivered during 2-year observation had a 10 times higher incidence rate of neuropathy $(p<0.001)$, compared to control subjects. In the long-term, however, the history of pregnancy does not seem to increase the risk of diabetes complications [11]. The presented results also suggest that neither the incidence nor the onset of neuropathy is related to parity. It correlates only with age, DM duration, age at diagnosis, average $\mathrm{HbA}_{1 \mathrm{c}}$ and systolic blood pressure. DM duration and mean $\mathrm{HbA}_{1 \mathrm{c}}$ from the course of the disease remained as neuropathy predictors in multivariate logistic regression analysis.

Cardiovascular complications. Little data exists concerning the impact of parity on macroangiopatic complications. Coronary artery disease is not a common complication observed during pregnancy as it usually develops later in life. Moreover, women with a history of myocardial infarction or suffering from angina that is difficult to stabilize are generally discouraged from becoming pregnant. A study by Hemachandra et al. showed that obstetric history had no influence on the incidence of coronary heart disease, neither in the short-term nor in later life [11]. Similarly, the current observation showed that none of cardiovascular complications was influenced by obstetric history, except for the rate of ischaemic heart disease. Nevertheless, it should be underlined once again that this entity was noted in only one nulliparous woman, therefore, one must be very cautious in drawing general conclusions from this statistical calculation. DM duration, as in the case of retinopathy, nephropathy and neuropathy, was the strongest factor affecting the risk for macrovascular complications. Logistic regression analysis, however, did not confirm this relationship.

\section{CONCLUSIONS}

The presented data indicate that there is no difference in the incidence of chronic diabetes complications between parous women and the subjects who were never pregnant. Observations of many authors suggest that any noted progression of diabetic complications are transitional and tend to remit after delivery. Therefore, it seems that thanks to intensive medical surveillance and appropriate medical interventions during pregnancy, women with types 1 diabetes mellitus may contemplate pregnancy without any concern for their condition in the long-term.

\section{Acknowledgements}

This study was supported by Grant No. 503-1-107-02 from the Medical University of Lodz, Poland.

\section{REFERENCES}

1. Loukovaara S, Immonen I, Teramo KA, Kaaja R. Progression of retinopathy during pregnancy in type 1 diabetic women treated with insulin lispro. Diabetes Care 2003; 26(4): 1193-8.

2. Wender-Ozegowska E, Zawiejska A, Pietryga M, Zozulińska D, Wierusz-Wysocka B, Chmaj K et al. Effect of pregnancy on diabetic vascular complications. Ginekol Pol. 2004; 75(5): 342-51.

3. Kaaja R. Vascular complications in diabetic pregnancy. Thromb Res. 2011; 127 Suppl 3: S53-5.

4. Sunness JS. The pregnant woman's eye. Surv Ophtalmol 1988; 32: 219-238.

5. Rosenn B, Miodovnik M, Kranias G, Khoury J, Combs CA, Mimouni F et al. Progression of diabetic retinopathy in pregnancy. Am J Obstet Gynecol. 1992; 166: 1214-8.

6. The Diabetes Control and Complications Trial Research Group. Effect of pregnancy on microvascular complications in the diabetes control and complications trial. Diabetes Care 2000; 23: 1084-1091.

7. Chang S, Fuhrmann M, Jovanovich L. The diabetes in early pregnancy study group (DIEP). Pregnancy, retinopathy normoglycemia. A preliminary analysis. Diabetes 1985; 35: 3A.

8. Temple RC, Aldridge VA, Sampson MJ, Greenwood RH, Heyburn PJ, Glenn A. Impact of pregnancy on the progression of diabetic retinopathy in Type 1 diabetes. Diabet Med. 2001; 18(7): 573-7.

9. Moloney JB, Drury MI. The effect of pregnancy on the natural course of diabetic retinopathy. Am J Ophthalmol. 1982; 93(6): 745-56.

10. Dibble CM, Kochenour NK, Worley RJ, Tyler FH, Swartz M. Effect of pregnancy on diabetic retinopathy. Obstet Gynecol. 1982; 59(6): 699-704.

11. Hemachandra A, Ellis D, Lloyd CE, Orchard TJ. The influence of pregnancy on IDDM complications. Diabetes Care 1995; 18(7): 950-4. 
12. Proniewska-Skretek E, Zalewska R, Kraśnicki P, Zarzycka B, Zarzycki $\mathrm{W}$, Mariak Z et al. The influence of pregnancy on progression of diabetic retinopathy. Klin Oczna 2007; 109(7-9): 308-11.

13. Rasmussen KL, Laugesen CS, Ringholm L, Vestgaard M, Damm P, Mathiesen ER. Progression of diabetic retinopathy during pregnancy in women with type 2 diabetes. Diabetologia 2010; 53(6): 1076-83.

14. Kaaja R, Sjoberg L, Hellsted T, Immonen I, Sane T, Teramo K. Longterm effects of pregnancy on diabetic complications. Diabet Med. 1996; 13: 165-169.

15. Jovanovic R, Jovanovic L. Obstetric management when normoglycemia is maintained in diabetic pregnant women with vascular compromise. Am J Obstet Gynecol. 1984; 149: 617-23.
16. Gordon M, Landon MB, Samuels P, Hissrich S, Gabbe SG. Perinatal outcome and long-term followup associated with modern management of diabetic nephropathy (Class F) Obstet Gynecol. 1996; 87: 401-9.

17. Young EC, Pires ML, Marques LP, de Oliveira JE, Zajdenverg L. Effects of pregnancy on the onset and progression of diabetic nephropathy and of diabetic nephropathy on pregnancy outcomes. Diabetes Metab Syndr. 2011; 5(3): 137-42.

18. Miodovnik M, Rosenn BM, Khoury JC, Grigsby JL, Siddiqi TA. Does pregnancy increase the risk for development and progression of diabetic nephropathy? Am J Obstet Gynecol. 1996; 174(4): 1180-9; discussion 1189-91.

19. Rossing K, Jacobsen P, Hommel E, Mathiesen E, Svenningsen A, Rossing $\mathrm{P}$ et al. Pregnancy and progression of diabetic nephropathy. Diabetologia 2002; 45(1): 36-41. 